\title{
Complete genome sequence of the gliding, heparinolytic Pedobacter saltans type strain $\left(113^{\mathrm{T}}\right)$
}

\author{
Konstantinos Liolios ${ }^{1}$, Johannes Sikorski ${ }^{2}$, Meagan Lu ${ }^{3}$, Matt Nolan ${ }^{1}$, Alla Lapidus ${ }^{1}$, Susan \\ Lucas $^{1}$, Nancy Hammon ${ }^{1}$, Shweta Deshpande ${ }^{1}$, Jan-Fang Cheng ${ }^{1}$, Roxanne Tapia ${ }^{1,3}$, Cliff \\ Han $^{1,3}$, Lynne Goodwin ${ }^{1,3}$, Sam Pitluck ${ }^{1}$, Marcel Huntemann ${ }^{1}$, Natalia Ivanova ${ }^{1}$, Ioanna \\ Pagani $^{1}$, Konstantinos Mavromatis ${ }^{1}$, Galina Ovchinikova ${ }^{1}$, Amrita Pati ${ }^{1}$, Amy Chen ${ }^{4}$, Krishna \\ Palaniappan $^{4}$, Miriam Land ${ }^{1,5}$, Loren Hauser ${ }^{1,5}$, Evelyne-Marie Brambilla ${ }^{2}$, Oleg \\ Kotsyurbenko ${ }^{6,7}$, Manfred Rohde ${ }^{8}$, Brian J. Tindall ${ }^{2}$, Birte Abt $^{2}$, Markus Göker ${ }^{2}$, John C. \\ Detter $^{1,3}$, Tanja Woyke ${ }^{1}$, James Bristow ${ }^{1}$, Jonathan A. Eisen ${ }^{1,9}$, Victor Markowitz ${ }^{4}$, Philip \\ Hugenholtz ${ }^{1,10}$, Hans-Peter Klenk ${ }^{2}$, and Nikos C. Kyrpides ${ }^{1 *}$ \\ ${ }^{1}$ DOE Joint Genome Institute, Walnut Creek, California, USA \\ ${ }^{2}$ DSMZ - German Collection of Microorganisms and Cell Cultures GmbH, Braunschweig, \\ Germany \\ ${ }^{3}$ Los Alamos National Laboratory, Bioscience Division, Los Alamos, New Mexico, USA \\ ${ }^{4}$ Biological Data Management and Technology Center, Lawrence Berkeley National \\ Laboratory, Berkeley, California, USA \\ ${ }^{5}$ Oak Ridge National Laboratory, Oak Ridge, Tennessee, USA \\ ${ }^{6}$ Technical University of Braunschweig, Institute for Microbiology, Braunschweig, Germany \\ ${ }^{7}$ Lomonosov Moscow State University, Biological Department, Moscow, Russia \\ ${ }^{8}$ HZI - Helmholtz Centre for Infection Research, Braunschweig, Germany \\ ${ }^{9}$ University of California Davis Genome Center, Davis, California, USA \\ ${ }^{10}$ Australian Centre for Ecogenomics, School of Chemistry and Molecular Biosciences, The \\ University of Queensland, Brisbane, Australia \\ *Corresponding author: Nikos C. Kyrpides
}

Keywords: strictly aerobic, gliding motility, Gram-negative, heparinolytic, mesophilic, chemoorganotrophic, Sphingobacteriaceae, GEBA

\begin{abstract}
Pedobacter saltans Steyn et al. 1998 is one of currently 32 species in the genus Pedobacter within the family Sphingobacteriaceae. The species is of interest for its isolated location in the tree of life. Like other members of the genus $P$. saltans is heparinolytic. Cells of $P$. saltans show a peculiar gliding, dancing motility and can be distinguished from other Pedobacter strains by their ability to utilize glycerol and the inability to assimilate D-cellobiose. The genome presented here is only the second completed genome sequence of a type strain from a member of the family Sphingobacteriaceae to be published. The 4,635,236 bp long genome with its 3,854 protein-coding and 67 RNA genes consists of one chromosome, and is a part of the Genomic Encyclopedia of Bacteria and Archaea project.
\end{abstract}

\section{Introduction}

Strain $113^{\mathrm{T}}$ (= DSM $12145=$ LMG $10337=$ NBRC 100064 ) is the type strain of the species Pedobacter saltans [1], one of currently 32 validly named species in the genus Pedobacter [2]. We prefer to use here the strain designation ' 113 ' as originally published by Steyn et al. in 1992 [3] and as also shown in the LMG online catalogue [4] and in the StrainInfo database [5] over the designation 'LMG $10337^{\prime}$ ' which was later used for the description of the species by the same authors [1]. The genus name is derived from the Latinized Greek word 'pedon' meaning 'the ground, earth' and the NeoLatin word 'bacter' meaning 'rod', yielding 'Pedobacter', the 'rod from soil' [1]. The species epithet is derived from the Latin word 'saltare' meaning 'to dance', yielding 'saltans', referring to the gliding motility of the strain' [1]. P. saltans strain $113^{\mathrm{T}}$ was isolated from soil in Iceland; 
Liolios et al.

several more strains belonging to the species were isolated from soil in Iceland, Belgium (Brussels) and Germany (Rüdesheim) [1]. Members of the genus Pedobacter were isolated from various environments including different soils [1,6-10], water [11-13], a nitrifying inoculum [14], glaciers $[15,16]$, fish [1] and compost [17]. Here we present a summary classification and a set of features for P. saltans strain $113^{\mathrm{T}}$, together with the description of the complete genome sequence and the genome annotation.

\section{Classification and features}

A representative genomic 16S rRNA sequence of strain $113^{\mathrm{T}}$ was compared using NCBI BLAST $[18,19]$ under default settings (e.g., considering only the high-scoring segment pairs (HSPs) from the best 250 hits) with the most recent release of the Greengenes database [20] and the relative frequencies of taxa and keywords (reduced to their stem [21]) were determined, weighted by BLAST scores. The most frequently occurring genera were Pedobacter (53.4\%), Sphingobacterium (33.3\%), Mucilaginibacter (5.0\%), Flavobacterium (4.1\%) and 'Sphingoterrabacterium' (2.1\%) (95 hits in total). Regarding the two hits to sequences from members of the species, the average identity within HSPs was 99.7\%, whereas the average coverage by HSPs was $97.6 \%$. Regarding the 20 hits to sequences from other members of the genus, the average identity within HSPs was $92.8 \%$, whereas the average coverage by HSPs was $84.1 \%$. Among all other species, the one yielding the highest score was Pedobacter lentus (EF446146), which corresponded to an identity of $93.2 \%$ and an HSP coverage of $93.4 \%$. (Note that the Greengenes database uses the INSDC (= EMBL/NCBI/DDBJ) annotation, which is not an authoritative source for nomenclature or classification.) The highestscoring environmental sequence was HM008274 ('anodic biomass air-cathode single chamber microbial fuel cell clone 9week.anode.2'), which showed an identity of $94.6 \%$ and an HSP coverage of $83.8 \%$. The most frequently occurring keywords within the labels of environmental samples which yielded hits were 'skin' (8.6\%), 'fossa' (4.2\%), 'poplit' (2.2\%), 'soil' (2.2\%) and 'forearm, volar' $(2.0 \%)$ (152 hits in total). Interestingly, several of the most frequent keywords relate to a mammalian or clinical habitats, which may allude to some yet unknown ecological features of
P. saltans, taking into account that all known isolates are from soil in different countries [1]. However, environmental samples which yielded hits of a higher score than the highest scoring species were not found.

Figure 1 shows the phylogenetic neighborhood of $P$. saltans in a $16 \mathrm{~S}$ rRNA based tree. The sequences of the four $16 \mathrm{~S}$ rRNA gene copies in the genome differ from each other by up to one nucleotide, and differ by up to three nucleotides from the previously published $16 \mathrm{~S}$ rRNA sequence (AJ438173).

The cells of $P$. saltans are short rods $(0.5 \times 0.7-1.0$ $\mu \mathrm{m}$ ) with rounded or slightly tapering ends (Figure 2) [1]. Three of the four strains belonging to $P$. saltans were described as being motile via gliding [1]. P. saltans cells strain Gram-negative and are non-spore-forming (Table 1 ). Strain $113^{\mathrm{T}}$ is strictly aerobic and chemoorganotrophic [1]. Colonies on modified TSA are smooth, light yellow to yellow, translucent, round, 2-5 $\mathrm{mm}$ in diameter, convex to slightly umbonate with entire margins [1]. On nutrient agar colonies are smooth, yellow, round, 2-4 $\mathrm{mm}$ in diameter, convex with entire to scalloped margins [1]. The temperature range for growth is normally between $5^{\circ} \mathrm{C}$ and $30^{\circ} \mathrm{C}$ [1]. The biochemical features and antibiotic resistance of $P$. saltans has been described previously [1]. Strain $113^{\mathrm{T}}$ produces acetoin from sodium pyruvate, degrades chondroitin sulfate and hydrolyzes aesculin. It grows on heparin, which is degraded by inducible enzymes. Good growth occurs on nutrient agar or on regular or modified TSA. P. saltans does not produce $\mathrm{H}_{2} \mathrm{~S}$ from thiosulfate and does not grow on MacConkey agar [1]. P. saltans can be differentiated phenotypically from other Pedobacter species by its inability to assimilate D-cellobiose and the ability to utilize glycerol. The organism does not reduce nitrate [1].

\section{Chemotaxonomy}

The cell wall of the members of the genus Pedobacter contain sphingolipids and menaquinone- 7 as the predominant menaquinone system [11-13]. Strain $113^{\mathrm{T}}$ contains the following fatty acids: iso$\mathrm{C}_{15: 0}(31.4 \%), \mathrm{C}_{16: 1 \omega 7 \mathrm{c}}(19.6 \%)$, iso- $\mathrm{C}_{17: 0 \text { 3-он }}(12.7 \%)$, iso- $\mathrm{C}_{15: 0}$ 2-он $(8.9 \%)$, iso- $\mathrm{C}_{17: 1 \omega 9 \mathrm{c}}(6.6 \%), \mathrm{C}_{16: 0}(4.0 \%)$, anteiso- $\mathrm{C}_{15: 0} \quad(2.9 \%)$, iso- $\mathrm{C}_{15: 0}$ 3-он $\quad(2.8 \%), \mathrm{C}_{15: 0}$ (1.4\%), $\mathrm{C}_{15: 1 \omega 6 \mathrm{c}}(1.4 \%)$, and $\mathrm{C}_{16: 1 \omega 7 \mathrm{c}}(19.6 \%)$ which are acids typical of the genus. It also contains traces

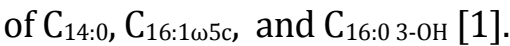




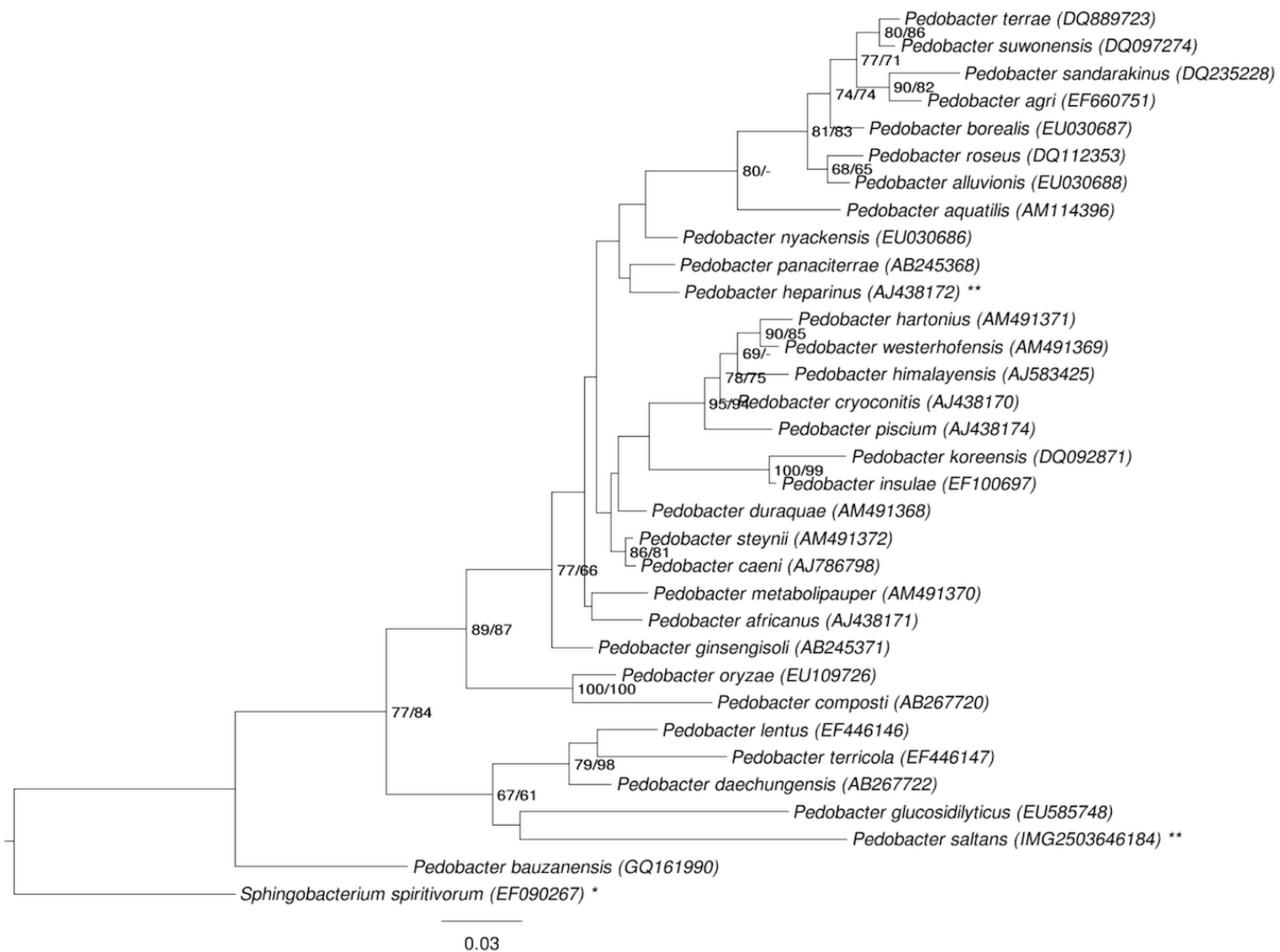

Figure 1. Phylogenetic tree highlighting the position of $P$. saltans relative to the other type strains within the genus $P e-$ dobacter. The tree was inferred from 1,402 aligned characters $[22,23]$ of the $16 \mathrm{~S}$ rRNA gene sequence under the maximum-likelihood (ML) criterion [24] and rooted with the type strain of the type species of the family Sphingobacteriaceae [25]. The branches are scaled in terms of the expected number of substitutions per site. Numbers adjacent to the branches are support values from $550 \mathrm{ML}$ bootstrap replicates [26] (left) and from 1,000 maximum-parsimony bootstrap replicates [27] (right) if larger than 60\%. Lineages with type strain genome sequencing projects registered in GOLD [28] as unpublished are marked with one asterisk, those listed as published with two asterisks [29]. Note that the taxon selection used in this figure does not allow conclusions about the monophyly of the genus Pedobacter. In an expanded analysis also including the genera Mucilaginibacter and Nubsella (data not shown), neither the Kishino-Hasegawa test as implemented in PAUP* [27] in conjunction with the maximum-parsimony criterion nor the Shimodaira-Hasegawa test as implemented in RAxML [24] in conjunction with the ML criterion indicated a significant difference between the respective globally best tree and the best tree after constraining for the monophyly of all four genera. (See, e.g. chapter 21 in [30] for an in-depth description of such paired-site tests.)

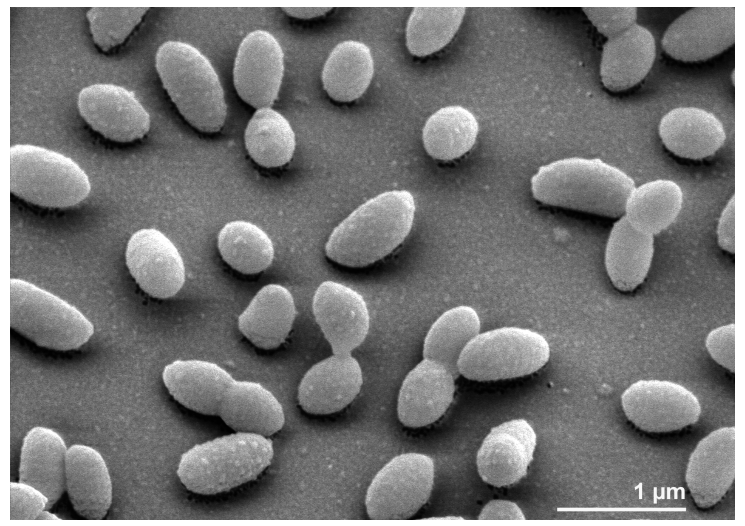

Figure 2. Scanning electron micrograph of $\mathrm{P}$. saltans strain $113^{\top}$. 
Table 1. Classification and general features of $P$. saltans strain $113^{\top}$ according to the MIGS recommendations [31] and the NamesforLife database [2]

\begin{tabular}{|c|c|c|c|}
\hline MIGS ID & Property & Term & Evidence code \\
\hline & \multirow{8}{*}{ Current classification } & Domain Bacteria & TAS [32] \\
\hline & & Phylum "Bacteroidetes" & TAS [33] \\
\hline & & Class "Sphingobacteria" & TAS [34] \\
\hline & & Order "Sphingobacteriales" & TAS [34] \\
\hline & & Family Sphingobacteriaceae & TAS [1] \\
\hline & & Genus Pedobacter & TAS $[1,11,12,14]$ \\
\hline & & Species Pedobacter saltans & TAS [1] \\
\hline & & Type strain 113 & TAS $[1,3]$ \\
\hline & Gram stain & negative & TAS [1] \\
\hline & Cell shape & short rods with rounded, slightly tapering ends & TAS [1] \\
\hline & Motility & gliding & TAS [1] \\
\hline & Sporulation & none & TAS [1] \\
\hline & Temperature range & $5^{\circ} \mathrm{C}-30^{\circ} \mathrm{C}$ & TAS [1] \\
\hline & Optimum temperature & not reported & \\
\hline & Salinity & not reported & \\
\hline \multirow[t]{3}{*}{ MIGS-22 } & Oxygen requirement & strictly aerobic & TAS [1] \\
\hline & Carbon source & carbohydrates, some alcohols and glycosides & TAS [1] \\
\hline & Energy metabolism & chemoorganotroph & TAS [1] \\
\hline MIGS-6 & Habitat & soil & TAS [1] \\
\hline MIGS-15 & Biotic relationship & free-living & NAS \\
\hline \multirow[t]{3}{*}{ MIGS-14 } & Pathogenicity & none & NAS \\
\hline & Biosafety level & 1 & TAS [35] \\
\hline & Isolation & soil & TAS [3] \\
\hline MIGS-4 & Geographic location & Iceland & TAS $[1,3]$ \\
\hline MIGS-5 & Sample collection time & 1992 or before & TAS $[1,3]$ \\
\hline MIGS-4.1 & Latitude & not reported & \\
\hline MIGS-4.2 & Longitude & not reported & \\
\hline MIGS-4.3 & Depth & not reported & \\
\hline MIGS-4.4 & Altitude & not reported & \\
\hline
\end{tabular}

Evidence codes - TAS: Traceable Author Statement (i.e., a direct report exists in the literature); NAS: Nontraceable Author Statement (i.e., not directly observed for the living, isolated sample, but based on a generally accepted property for the species, or anecdotal evidence). These evidence codes are from of the Gene Ontology project [36].

\section{Genome sequencing and annotation Genome project history}

This organism was selected for sequencing on the basis of its phylogenetic position [37], and is part of the Genomic Encyclopedia of Bacteria and Archaea project [38]. The genome project is deposited in the Genome OnLine Database [28] and the complete genome sequence is deposited in GenBank. Sequencing, finishing and annotation were performed by the DOE Joint Genome Institute (JGI). A summary of the project information is shown in Table 2. 
Table 2. Genome sequencing project information

\begin{tabular}{lll}
\hline MIGS ID & Property & Term \\
\hline MIGS-31 & Finishing quality & Finished \\
MIGS-28 & Libraries used & Tree genomic libraries: one 454 pyrosequence standard library, one \\
MIGS-29 & Sequencing platforms & 454 PE library (7.7 kb insert size), one Illumina library \\
MIGS-31.2 & Sequencing coverage & $645.0 \times$ Illumina; $19.5 \times$ pyrosequence \\
MIGS-30 & Assemblers & Newbler version 2.3, Velvet version 0.7.63, phrap version SPS - 4.24 \\
MIGS-32 & Gene calling method & Prodigal 1.4, GenePRIMP \\
& INSDC ID & CP002545 \\
& Genbank Date of Release & March 2, 2011 \\
& GOLD ID & Gc01673 \\
& NCBI project ID & 49337 \\
& Database: IMG-GEBA & 649633082 \\
MIGS-13 & Source material identifier & DSM 12145 \\
& Project relevance & Tree of Life, GEBA \\
\hline
\end{tabular}

\section{Growth conditions and DNA isolation}

P. saltans $113^{\mathrm{T}}$ (DSM 12145), was grown in DSMZ medium 605 (Nutrient agar (Oxoid CM3)) [39] at $28^{\circ} \mathrm{C}$. DNA was isolated from $0.5-1 \mathrm{~g}$ of cell paste using Jetflex Genomic DNA Purification Kit (GENOMED 600100), modified by 1 hour incubation at $58^{\circ} \mathrm{C}$ with $20 \mu \mathrm{l}$ proteinase for improved cell lysis. DNA is available through the DNA Bank Network [40].

\section{Genome sequencing and assembly}

The genome was sequenced using a combination of Illumina and 454 sequencing platforms. All general aspects of library construction and sequencing can be found at the JGI website [41]. Pyrosequencing reads were assembled using the Newbler assembler (Roche). The initial Newbler assembly consisting of 44 contigs in two scaffolds was converted into a phrap [42] assembly by making fake reads from the consensus, to collect the read pairs in the 454 paired end library. Illumina sequencing data $(6,233.8 \mathrm{Mb})$ was assembled with Velvet [43] and the consensus sequences were shredded into $1.5 \mathrm{~kb}$ overlapped fake reads and assembled together with the 454 data. The 454 draft assembly was based on $112.7 \mathrm{Mb} 454 \mathrm{draft}$ data and all of the 454 paired end data. Newbler parameters are -consed -a 50 -l 350 -g -m -ml 20. The Phred/Phrap/Consed software package [42] was used for sequence assembly and quality assessment in the subsequent finishing process. After the shotgun stage, reads were assembled with parallel phrap (High Performance Software, LLC).
Possible mis-assemblies were corrected with gapResolution [41], Dupfinisher [44], or sequencing cloned bridging PCR fragments with subcloning. Gaps between contigs were closed by editing in Consed, by PCR and by Bubble PCR primer walks (J.-F. Chang, unpublished). A total of 205 additional reactions were necessary to close gaps and to raise the quality of the finished sequence. Illumina reads were also used to correct potential base errors and increase consensus quality using a software Polisher developed at JGI [45]. The error rate of the completed genome sequence is less than 1 in 100,000. Together, the combination of the Illumina and 454 sequencing platforms provided $664.5 \times$ coverage of the genome. The final assembly contained 205,963 pyrosequence and $82,382,711$ Illumina reads.

\section{Genome annotation}

Genes were identified using Prodigal [46] as part of the Oak Ridge National Laboratory genome annotation pipeline, followed by a round of manual curation using the JGI GenePRIMP pipeline [47]. The predicted CDSs were translated and used to search the National Center for Biotechnology Information (NCBI) non-redundant database, UniProt, TIGR-Fam, Pfam, PRIAM, KEGG, COG, and InterPro databases. Additional gene prediction analysis and functional annotation was performed within the Integrated Microbial Genomes - Expert Review (IMG-ER) platform [48]. 


\section{Genome properties}

The genome consists of a 4,635,236 bp long chromosome with a $\mathrm{G}+\mathrm{C}$ content of $36.6 \%$ (Figure 3 and Table 3). Of the 3,921 genes predicted, 3,854 were protein-coding genes, and 67 RNAs; 62 pseudogenes were also identified. The majority of the protein-coding genes $(64.8 \%)$ were assigned a putative function while the remaining ones were annotated as hypothetical proteins. The distribution of genes into COGs functional categories is presented in Table 4.

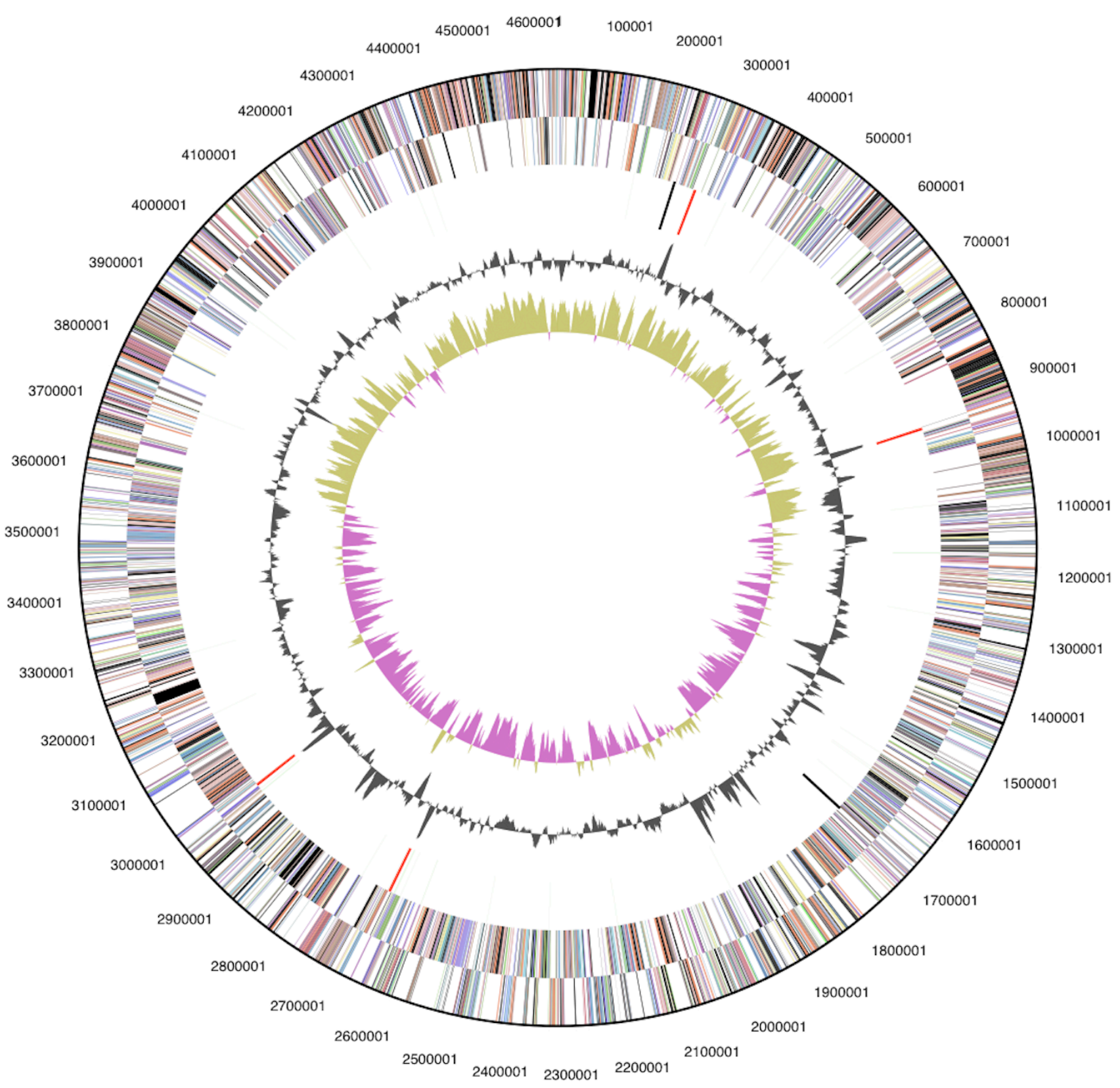

Figure 3. Graphical circular map of genome. From outside to the center: Genes on forward strand (color by COG categories), Genes on reverse strand (color by COG categories), RNA genes (tRNAs green, rRNAs red, other RNAs black), GC content, GC skew. 
Table 3. Genome Statistics

\begin{tabular}{lrr}
\hline Attribute & Value & \% of Total \\
\hline Genome size (bp) & $4,635,236$ & $100.00 \%$ \\
DNA coding region (bp) & $4,149,395$ & $89.52 \%$ \\
DNA G+C content (bp) & $1,695,689$ & $36.58 \%$ \\
Number of replicons & 1 & \\
Extrachromosomal elements & 0 & \\
Total genes & 3,921 & $100.00 \%$ \\
RNA genes & 67 & $1.71 \%$ \\
rRNA operons & 4 & \\
Protein-coding genes & 3,854 & $98.29 \%$ \\
Pseudo genes & 62 & $1.58 \%$ \\
Genes with function prediction & 2,539 & $64.75 \%$ \\
Genes in paralog clusters & 87 & $2.22 \%$ \\
Genes assigned to COGs & 2,644 & $67.43 \%$ \\
Genes assigned Pfam domains & 2,757 & $70.31 \%$ \\
Genes with signal peptides & 1,646 & $41.98 \%$ \\
Genes with transmembrane helices & 898 & $22.90 \%$ \\
CRISPR repeats & 0 & \\
\hline
\end{tabular}

Table 4. Number of genes associated with the general COG functional categories

\begin{tabular}{crrl}
\hline Code & value & \%age & Description \\
\hline J & 158 & 5.5 & Translation, ribosomal structure and biogenesis \\
A & 0 & 0.0 & RNA processing and modification \\
K & 175 & 6.1 & Transcription \\
L & 142 & 5.0 & Replication, recombination and repair \\
B & 1 & 0.0 & Chromatin structure and dynamics \\
D & 26 & 0.9 & Cell cycle control, cell division, chromosome partitioning \\
Y & 0 & 0.0 & Nuclear structure \\
V & 55 & 1.9 & Defense mechanisms \\
T & 146 & 5.1 & Signal transduction mechanisms \\
M & 278 & 9.7 & Cell wall/membrane/envelope biogenesis \\
N & 11 & 0.4 & Cell motility \\
Z & 0 & 0.0 & Cytoskeleton \\
W & 0 & 0.0 & Extracellular structures \\
U & 47 & 1.6 & Intracellular trafficking, secretion, and vesicular transport \\
O & 106 & 3.7 & Posttranslational modification, protein turnover, chaperones \\
C & 157 & 5.5 & Energy production and conversion \\
G & 282 & 9.8 & Carbohydrate transport and metabolism \\
E & 172 & 6.0 & Amino acid transport and metabolism \\
F & 69 & 2.4 & Nucleotide transport and metabolism \\
H & 128 & 4.5 & Coenzyme transport and metabolism \\
I & 86 & 3.0 & Lipid transport and metabolism \\
P & 195 & 6.8 & Inorganic ion transport and metabolism \\
Q & 41 & 1.4 & Secondary metabolites biosynthesis, transport and catabolism \\
R & 355 & 12.4 & General function prediction only \\
S & 238 & 8.3 & Function unknown \\
- & 1,277 & 32.6 & Not in COGs \\
\hline
\end{tabular}




\section{Insights into the genome sequence}

An estimate of the overall similarity between $\mathrm{Pe}$ dobacter heparinus and P. saltans [1] was generated with the GGDC-Genome-to-Genome Distance Calculator $[49,50]$. This system calculates the distances by comparing the genomes to obtain highscoring segment pairs (HSPs) and interfering distances from a set of three formulae (1, HSP length
/ total length; 2, identities / HSP length; 3, identities / total length). The comparison of $P$. heparinus and $P$. saltans revealed that an average of only $4.7 \%$ of the two genomes are covered with HSPs. The identity within these HSPs was $82.3 \%$, whereas the identity over the whole genome was $3.8 \%$.

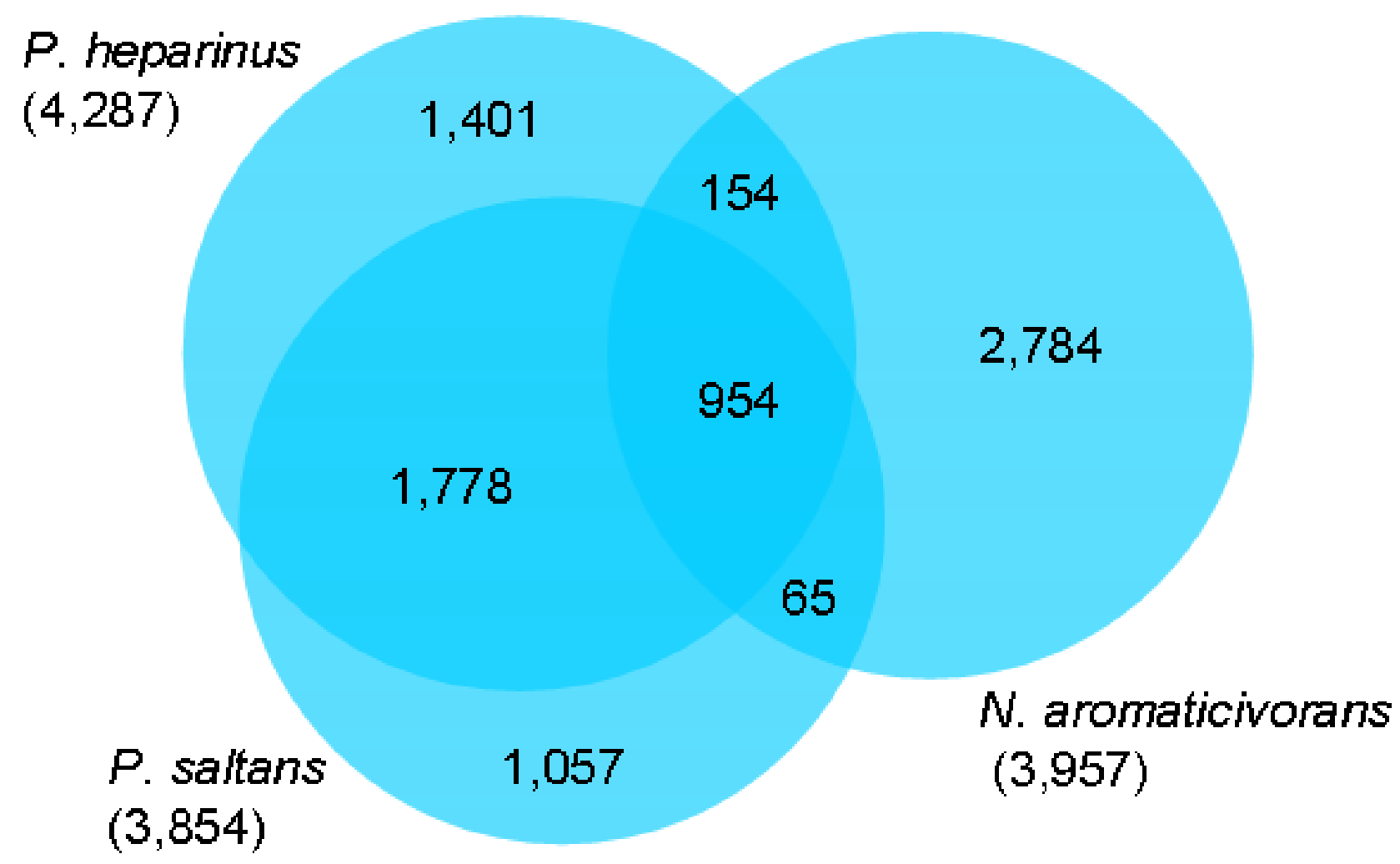

Figure 4. Venn diagram depicting the intersections of protein sets (total number of derived protein sequences in parentheses) of $\mathrm{P}$. heparinus, P. saltans and N. aromaticivorans.

The fraction of shared genes in the genomes of $P$. heparinus, $P$. saltans and Novosphingobium aromaticivorans [51] is shown in a Venn diagram (Figure 4). The phyogentically distant reference genome of $N$. aromaticivorans was selected based on its similar genome size and due to a lack of complete reference type strain genomes from the Sphingobacteriaceae. The numbers of pairwise shared genes were calculated with the phylogenetic profiler function of the IMG ER platform [48]. The homologous genes within the genomes were detected with a maximum E-value of $10^{-5}$ and a minimum identity of $30 \%$. Only about one quarter of all genes (954 genes) are shared by all three genomes, whereas the two Pedobacter species share 2,732 genes, corresponding to $63.7 \%(P$. heparinus) and $70.9 \%$ (P. saltans) of their genes. The pairwise comparison of $N$. aromaticivorans with the two Pedobacter species revealed only 154 (P. heparinus) and 65 (N. aromaticivorans) homologous genes (Figure 4).

Among those genes that are shared by the three genomes, are those which might be responsible for the yellow color of the organisms. These genes encode enzymes that are involved in the synthesis of carotenoids. Biosynthesis of carotenoids starts with geranylgeranyl pyrophosphate synthases combining farnesyl pyrophosphate with 
$\mathrm{C}_{5}$ isoprenoid units to $\mathrm{C}_{20}$-molecules, geranylgeranyl pyrophosphate. The phytoene synthase catalyzes the condensation of two geranylgeranyl pyrophosphate molecules followed by the removal of diphosphate and a proton shift leading to the formation of phytoene. Sequential desaturation steps are catalyzed by phytoene desaturase followed by cyclization of the ends of the molecules catalyzed by the lycopene cyclase [52]. Genes encoding lycopene cyclases (Phep_2088, Pedsa_2222, Saro_1817) and phytoene synthases (Phep_2092, Pedsa_2218, Saro_1814) were identified in the genomes. In the two Pedobacter species, genes coding for phytoene desaturases (Phep_2093, Pedsa_2217) were also identified. A carotene hydroxylase gene (Saro_1168) was only identified in the genome of $N$. aromaticivorans.

As the two Pedobacter species are known for their ability to degrade heparin, it is not surprising that the genomes encode several heparinase encoding

\section{Acknowledgements}

We would like to gratefully acknowledge the help of Helga Pomrenke (DSMZ) for growing P. saltans cultures. This work was performed under the auspices of the US Department of Energy Office of Science, Biological and Environmental Research Program, and by the University of California, Lawrence Berkeley National Laboratory under contract No. DE-AC02-05CH11231,

\section{References}

1. Steyn PL, Segers P, Vancanneyt M, Sandra P, Kersters K, Joubert JJ. Classification of heparinolytic bacteria into a new genus, Pedobacter, comprising four species: Pedobacter heparinus comb. nov., Pedobacter piscium comb. nov., Pedobacter africanus sp. nov. and Pedobacter saltans sp. nov. Proposal of the family Sphingobacteriaceae. Int J Syst Bacteriol 1998; 48:165-177. PubMed doi:10.1099/00207713-48-1-165

2. Garrity G. NamesforLife. BrowserTool takes expertise out of the database and puts it right in the browser. Microbiol Today 2010; 37:9.

3. Steyn PL, Pot B, Segers P, Kesters K, Joubert JJ. Some novel aerobic heparin-degrading bacterial isolates. Syst Appl Microbiol 1992; 15:137-143.

4. BCCM/LMG bacteria catalogue: http://bccm.belspo.be/db/lmg_species_details.php

5. StrainInfo website: http://www.straininfo.net/strains/45464

6. Jeon Y, Kim JM, Park JH, Lee SH, Seong CN, Lee SS, Jeon CO. Pedobacter oryzae sp.nov., isolated genes: seven ( $P$. saltans) and five ( $P$. heparinus) heparinases, were identified, whereas $N$. aromaticivorans encodes only one heparinase.

Fucoidan degradation was not determined experimentally, but is assumed as both $P$. saltans and $P$. heparinus have genes for eleven and ten $\alpha$ fucosidases respectively. In addition, 12 ( $P$. saltans) and 18 ( $P$. heparinus) $\alpha$-sulfatases genes were identified, whereas $N$. aromaticivorans contains only five $\alpha$-sulfatases and no $\alpha$-fucosidase genes. Experimental evidence for the fucoidan hydrolysis in Pedobacter has not been found, but for Mucilaginibacter paludis and M. gracilis, which are also members of the family Sphingobacteriaceae, have been experimentally confirmed to exhibit fucoidan degradation [53]. Moreover, Sakai et al. [54] reported the existence of intracellular $\alpha$-Lfucosidases and sulfatases, which enable ' $F$. fucoidanolyticus' to degrade fucoidan.

Lawrence Livermore National Laboratory under Contract No. DE-AC52-07NA27344, and Los Alamos National Laboratory under contract No. DE-AC0206NA25396, UT-Battelle and Oak Ridge National Laboratory under contract DE-AC05-000R22725, as well as German Research Foundation (DFG) INST 599/1-2.

from rice paddy soil. Int / Syst Evol Microbiol 2009; 59:2491-2495. PubMed doi:10.1099/ijs.0.005710-0

7. Zhang DC, Schinner F, Margesin R. Pedobacter bauzanensis sp. nov., isolated from soil. Int J Syst Evol Microbiol 2010; 60:2592-2595. PubMed doi:10.1099/ijs.0.018903-0

8. Yoon JH, Kang SJ, Park S, Oh TK. Pedobacter lentus sp.nov. and Pedobacter terricola sp. nov., isolated from soil. Int J Syst Evol Microbiol 2007; 57:2089-2095. PubMed doi:10.1099/ijs.0.65146$\underline{0}$

9. Luo X, Wang Z, Dai J, Zhang L, Li J, Tang Y, Wang Y, Fang C. Pedobacter glucosidilyticus sp.nov., isolated from dry riverbed soil. Int I Syst Evol Microbiol 2010; 60:229-233. PubMed doi:10.1099/ijs.0.008060-0

10. Yali T, Wang Y, Ji S, Zhang K, Dai J, Zhang L, Peng F, Fang C. Pedobacter xinjiangensis sp. nov., from the Desert, Xinjiang. Int / Syst Evol Microbiol 2010; 20:397-402. 
Liolios et al.

11. Hwang CY, Choi DH, Cho BC. Pedobacter roseus sp. nov., isolated from a hypertrophic pond, and emended description of the genus Pedobacter. Int J Syst Evol Microbiol 2006; 56:1831-1836. PubMed doi:10.1099/ijs.0.64045-0

12. Gallego V, Garcia MT, Ventosa A. Pedobacter aquatilis sp. nov., isolated from drinking water, and emended description of the genus Pedobacter. Int J Syst Evol Microbiol 2006; 56:1853-1858. PubMed doi:10.1099/ijs.0.64176-0

13. Joung Y, Kim H, Joh K. Pedobacter yonginense sp.nov., isolated from a mesotrophic artificial lake in Korea. J Microbiol 2010; 48:536-540. PubMed doi:10.1007/s12275-010-0010-4

14. Vanparys B, Heylen K, Lebbe L, de Vos P. Pedobacter caeni sp. nov., a novel species isolated from a nitrifying inoculum. Int J Syst Evol Microbiol 2005; 55:1315-1318. PubMed doi:10.1099/ijs.0.63635-0

15. Margesin R, Spröer C, Schumann P, Schinner F. Pedobacter cryoconitis sp.nov., a facultative psychrophile from alpine glacier cryoconite. Int J Syst Evol Microbiol 2003; 53:1291-1296. PubMed doi:10.1099/ijs.0.02436-0

16. Shivaji S, Chaturvedi P, Reddy GSN, Suresh K. Pedobacter himalayensis sp.nov., from the Hamta glacier located in the Himalayan mountain ranges of India. Int J Syst Evol Microbiol 2005; 55:10831088. PubMed doi:10.1099/ijs.0.63532-0

17. Lee HG, Kim SG, Im WT, Oh HM, Lee ST. Pedobacter composti sp. nov., isolated from compost. Int I Syst Evol Microbiol 2009; 59:345-349. PubMed doi:10.1099/ijs.0.003061-0

18. Altschul SF, Gish W, Miller W, Myers E, Lipman D. Basic local alignment search tool. I Mol Biol 1990; 215:403-410. PubMed

19. Korf I, Yandell M, Bedell J. BLAST, O'Reilly, Sebastopol, 2003

20. DeSantis TZ, Hugenholtz P, Larsen N, Rojas M, Brodie EL, Keller K, Huber T, Dalevi D, Hu P, Andersen GL. Greengenes, a chimera-checked $16 \mathrm{~S}$ rRNA gene database and workbench compatible with ARB. Appl Environ Microbiol 2006;

72:5069-5072. PubMed doi:10.1128/AEM.03006-05

21. Porter MF. An algorithm for suffix stripping Program: electronic library and information systems 1980; 14:130-137.

22. Lee C, Grasso C, Sharlow MF. Multiple sequence alignment using partial order graphs. Bioinformat-
iCs 2002; 18:452-464. PubMed

doi:10.1093/bioinformatics/18.3.452

23. Castresana J. Selection of conserved blocks from multiple alignments for their use in phylogenetic analysis. Mol Biol Evol 2000; 17:540-552. $\underline{\text { PubMed }}$

24. Stamatakis A, Hoover P, Rougemont J. A rapid bootstrap algorithm for the RAxML web-servers. Syst Biol 2008; 57:758-771. PubMed doi:10.1080/10635150802429642

25. Fitch WM. Toward defining the course of evolution: minimum change for a specific tree topology. Syst Zool 1971; 20:406-416. doi:10.2307/2412116

26. Pattengale ND, Alipour M, Bininda-Emonds ORP, Moret BME, Stamatakis A. How many bootstrap replicates are necessary? Lect Notes Comput Sci 2009; 5541:184-200. doi:10.1007/978-3-642$\underline{02008-7 \quad 13}$

27. Swofford DL. PAUP*: Phylogenetic Analysis Using Parsimony (*and Other Methods), Version 4.0 b10. Sinauer Associates, Sunderland, 2002.

28. Liolios K, Chen IM, Mavromatis K, Tavernarakis N, Hugenholtz P, Markowitz VM, Kyrpides NC. The Genomes On Line Database (GOLD) in 2009: status of genomic and metagenomic projects and their associated metadata. Nucleic Acids Res 2010; 38:D346-D354. PubMed doi:10.1093/nar/gkp848

29. Han C, Spring S, Lapidus A, Glavina Del Rio T, Tice H, Copeland A, Cheng JF, Lucas S, Chen F, Nolan $\mathrm{M}$, et al. Complete genome sequence of Pedobacter heparinus type strain (HIM 762-3 ${ }^{\mathrm{T}}$ ). Stand Genomic Sci 2009; 1:54-62. PubMed doi:10.4056/sigs.22138

30. Felsenstein J. Inferring phylogenies. Sinauer Associates Inc., Sunderland, Massachusetts 2004.

31. Field D, Garrity G, Gray T, Morrison N, Selengut J, Sterk P, Tatusova T, Thomson N, Allen MJ, Angiuoli $S V$, et al. The minimum information about a genome sequence (MIGS) specification. Nat Biotechnol 2008; 26:541-547. PubMed doi:10.1038/nbt1360

32. Woese CR, Kandler O, Wheelis ML. Towards a natural system of organisms: proposal for the domains Archaea, Bacteria, and Eucarya. Proc Natl Acad Sci USA 1990; 87:4576-4579. PubMed doi:10.1073/pnas.87.12.4576

33. Garrity GM, Holt JG. The Road Map to the Manual. In: Garrity GM, Boone DR, Castenholz RW (eds), Bergey's Manual of Systematic Bacteriology, 
Second Edition, Volume 1, Springer, New York, 2001, p. 119-169.

34. Garrity GM, Holt JG. Taxonomic outline of the Archaea and Bacteria. In: Garrity GM, Boone DR, Castenholz RW (eds), Bergey's Manual of Systematic Bacteriology, Second Edition, Volume 1, Springer, New York, 2001, p. 155-166.

35. BAuA. Classification of bacteria and archaea in risk groups. TRBA 2010; 466:165.

36. Ashburner M, Ball CA, Blake JA, Botstein D, Butler H, Cherry JM, Davis AP, Dolinski K, Dwight SS, Eppig JT, et al. Gene Ontology: tool for the unification of biology. Nat Genet 2000; 25:25-29. PubMed doi:10.1038/75556

37. Klenk HP, Göker M. En route to a genome-based classification of Archaea and Bacteria? Syst Appl Microbiol 2010; 33:175-182. PubMed doi:10.1016/j.syapm.2010.03.003

38. Wu D, Hugenholtz P, Mavromatis K, Pukall R, Dalin E, Ivanova NN, Kunin V, Goodwin L, Wu $M$, Tindall BJ, et al. A phylogeny-driven genomic encyclopaedia of Bacteria and Archaea. Nature 2009; 462:1056-1060. PubMed doi:10.1038/nature08656

39. List of growth media used at DSMZ: http://www.dsmz.de/microorganisms/media_list.p hp.

40. Gemeinholzer B, Dröge G, Zetzsche H, Haszprunar G, Klenk HP, Güntsch A, Berendsohn WG, Wägele JW. The DNA Bank Network: the start from a German initiative. Biopreservation and Biobanking 2011; 9:51-55.

doi:10.1089/bio.2010.0029

41. JGI website. http://www.jgi.doe.gov

42. The Phred/Phrap/Consed software package. http://www.phrap.com

43. Zerbino DR, Birney E. Velvet: algorithms for de novo short read assembly using de Bruijn graphs. Genome Res 2008; 18:821-829. PubMed doi:10.1101/gr.074492.107

44. Han C, Chain P. Finishing repeat regions automatically with Dupfinisher. In: Proceeding of the 2006 international conference on bioinformatics \& computational biology. Arabnia HR, Valafar $\mathrm{H}$ (eds), CSREA Press. June 26-29, 2006: 141-146.

45. Lapidus A, LaButti K, Foster B, Lowry S, Trong S, Goltsman E. POLISHER: An effective tool for us- ing ultra short reads in microbial genome assembly and finishing. AGBT, Marco Island, FL, 2008.

46. Hyatt D, Chen GL, LoCascio PF, Land ML, Larimer FW, Hauser LJ. Prodigal: prokaryotic gene recognition and translation initiation site identification. BMC Bioinformatics 2010; 11:119. PubMed doi:10.1186/1471-2105-11-119

47. Pati A, Ivanova NN, Mikhailova N, Ovchinnikova G, Hooper SD, Lykidis A, Kyrpides NC. GenePRIMP: a gene prediction improvement pipeline for prokaryotic genomes. Nat Methods 2010; 7:455-457. PubMed doi:10.1038/nmeth.1457

48. Markowitz VM, Ivanova NN, Chen IMA, Chu K, Kyrpides NC. IMG ER: a system for microbial genome annotation expert review and curation. Bioinformatics 2009; 25:2271-2278. PubMed doi:10.1093/bioinformatics/btp393

49. Auch AF, von Jan M, Klenk HP, Göker M. Digital DNA-DNA hybridization for microbial species delineation by means of genome-to-genome sequence comparison. Stand Genomic Sci 2010; 2:117-134. PubMed doi:10.4056/sigs.531120

50. Auch AF, Klenk HP, Göker M. Standard operating procedure for calculating genome-to-genome distances based on high-scoring segment pairs. Stand Genomic Sci 2010; 2:142-148. PubMed doi:10.4056/sigs.541628

51. Takeuchi M, Hamama K, Hiraishi A. Proposal of the genus Sphingomonas sensu stricto and three new genera, Sphingobium, Novosphingobium and Sphingopyxis, on the basis of phylogenetic and chemotaxonomic analyses. Int I Syst Evol Microbiol 2001; 51:1405-1417. PubMed

52. Sandmann G. Carotenoid biosynthesis and biotechnological application. Arch Biochem Biophys 2001; 385:4-12. PubMed doi:10.1006/abbi.2000.2170

53. Pankratov TA, Tindall BJ, Liesack W, Dedysh SN. Mucilaginibacter paludis gen. nov., sp. nov. and Mucilaginibacter gracilis sp. nov., pectin-, xylanand laminarin-degrading members of the family Sphingobacteriaceae from acidic Sphagnum peat bog. Int I Syst Evol Microbiol 2007; 57:23492354. PubMed doi:10.1099/ijs.0.65100-0

54. Sakai T, Ishizuka K, Kato I. Isolation and characterization of fucoidan-degrading marine bacterium. Mar Biotechnol 2003; 5:409-416. PubMed doi:10.1007/s10126-002-0118-6 